Abril Denisse Álvarez Castro (1) 0000-0001-5441-7993

Juan Carlos Morales Luna D 0000-0003-1500-3725 Félix D. Sánchez Godoy* (1) 0000-0002-8764-5762

Universidad Nacional Autónoma de México. Facultad de Medicina Veterinaria y Zootecnia.

* Autor para correspondencia: Correo electrónico: spuma91@hotmail.com

\section{Feminización e hiperostosis asociadas a tumor de células de Sertoli en un periquito australiano (Melopsittacus undulatus)}

\section{Resumen}

Descripción del caso. Periquito australiano (Melopsittacus undulatus), macho de siete años de edad, cuyo motivo de consulta fue cambio de coloración en la cera y distensión abdominal.

Hallazgos clínicos. En el examen físico general se encontraron signos clínicos de feminización, disnea y distensión abdominal. Se realizaron estudios radiográficos en donde se observó un tejido de neoformación hepatogonado-renal e hiperostosis generalizada.

Tratamiento y evolución. Se instauró tratamiento paliativo. Debido a la pobre evolución del paciente, el propietario decidió solicitar la eutanasia.

Pruebas de laboratorio. En el estudio radiográfico se observó radiopacidad generalizada en huesos largos, un tejido de nueva formación en la zona hepatogonado-renal y desplazamiento visceral. En la necropsia, los testículos exhibían un proceso neoplásico y con la histopatología se identificó un tumor de células de Sertoli.

Relevancia clínica. El objetivo de este trabajo es describir un síndrome paraneoplásico caracterizado por feminización e hiperostosis generalizada asociado a un tumor de células de Sertoli primario de testículo, el cual debe ser considerado como un diagnóstico clínico diferencial en neoplasias intracelómicas en machos.

Palabras clave: síndrome paraneoplásico, feminización, hiperostosis, tumor de células de Sertoli, periquitos australianos, Melopsittacus undulatus.

Recibido: 2019-11-04 Aceptado: 2020-03-03 Publicado: 2020-06-04 en la página 11

(a) Derechos de autor: Abril Denisse Álvarez Castro 2020

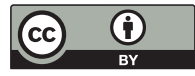

Una forma de citar este artículo:

Álvarez-Castro AD, Morales-Luna JC, Sánchez-Codoy FD. Feminización e hiperostosis asociadas a tumor de células de Sertoli en un periquito australiano (Melopsittacus undulatus). Clínica veterinaria: abordaje diagnóstico y terapéutico. 2020;6:e55202063. doi:10.22201/fmvz.23958766e.2020.6.55. 


\section{Feminization and hyperostosis associated with Sertoli cell tumor in Budgerigar (Melopsittacus undulatus)}

\section{Abstract}

Case report. Budgerigar (Melopsittacus undulatus), male, seven years old, with discoloration in the cere and abdominal distension.

Clinical findings. Physical examination revealed clinical signs of feminization, dyspnea, and abdominal distension. Radiographic studies were performed and hepatogonado-renal neoplasia and generalized hyperostosis were observed.

Treatment and evolution. Palliative treatment was established, due to the poor evolution of the patient, the owner decided to request euthanasia.

Laboratory tests. At necropsy, the testes exhibited a neoplastic process and with histopathology a Sertoli cell tumor was identified.

Clinical relevance. The objective of this work is describe a paraneoplastic syndrome characterized by feminization and generalized hyperostosis associated with a Sertoli cell tumor, which should be considered as a differential clinical diagnosis in intracelomic neoplasias in males.

Keywords: Paraneoplastic syndrome, feminization, hyperostosis, Sertoli cell tumor, Budgerigar, Melopsittacus undulatus. 


\section{Descripción del caso}

Se remitió para consulta al Hospital de Aves de la Facultad de Medicina Veterinaria y Zootecnia de la Universidad Nacional Autónoma de México (FMVZ-UNAM), un ave de la especie Melopsittacus undulatus, macho de siete años de edad aproximadamente. Su dieta estaba basada en alpiste compuesto (70 \%), manzana, pera, betabel, zanahoria, calabacita (30 \%) y agua de grifo. Convivía con una hembra de su misma especie en una jaula rectangular con bebedero y comedero de plástico, con perchas de madera de superficie lisa y de ramas de árbol de diferentes diámetros, se utilizaba periódico como sustrato y se realizaba la limpieza diariamente. El ave recibía baños con té de manzanilla tres veces por semana. No reportaron antecedentes médicos y el motivo de consulta era un abultamiento en abdomen y cambio de coloración en la cera de la rinoteca.

\section{Hallazgos clínicos e interpretación}

En el examen físico general el ave se observó alerta y responsiva, con un peso de 42 g y condición corporal 2/5; sin cambios aparentes en la frecuencia cardiaca y en los sonidos respiratorios, pero con disnea durante la manipulación; en la rinoteca la cera se apreció hipertrofiada y de color café (Figura 1). El abdomen distendido y firme al tacto, la piel de esta región, amarilla y con zonas aptéricas.

Con estos datos se establecieron los siguientes diagnósticos clínicos presuntivos: lipoma, neoplasia intracelómica o hernia abdominal. Se realizaron dos estudios radiográficos: uno ventrodorsal y otro laterolateral, en donde se observó radiopacidad generalizada en todos los huesos largos y un tejido de nueva formación en la zona hepatogonado-renal y desplazamiento de vísceras hacia caudal (Figura 2).

\section{Tratamiento y evolución}

Después de la revisión se hidrató con $3.99 \mathrm{~mL}$ de solución de lactato de sodio compuesto (solución Hartmann inyectable PISA) por vía subcutánea, y se envió a casa con diagnóstico presuntivo de hernia secundaria a lipoma, aunque no se descartó la posibilidad de una neoplasia en celoma. El tratamiento para casa fue suero oral en el agua de bebida ad libitum, suplemento de vitamina A (Vitafort, multivitamínico, polvo oral, PARFARM) 2000 UI en agua de bebida cada 24 horas por siete días, silimarina (Neocholal, cardo mariano, solución oral, ITALMEX) a $150 \mathrm{mg} / \mathrm{kg}$ PO cada 24 horas durante quince días y se indicó cambio de dieta (alpiste compuesto (30 \%) + linaza y chía (40 \%) más frutas y verduras (30\%).

El paciente acudió a revisión quince días después, se ingresó inmediatamente debido a que se encontraba en depresión, con las plumas erizadas, alas caídas y disnea. En la revisión clínica se detectó que el paciente se encontraba en condición corporal $1 / 5$ y peso corporal de 48 g, la región abdominal estaba más distendida y con eritema; la cloaca y las plumas timoneras tenían acumulación de heces. La propietaria refirió que, pese al estado del ave, no hubo alteración en el consumo de alimento.

Se decidió hospitalizar al paciente y se estableció el siguiente tratamiento: 


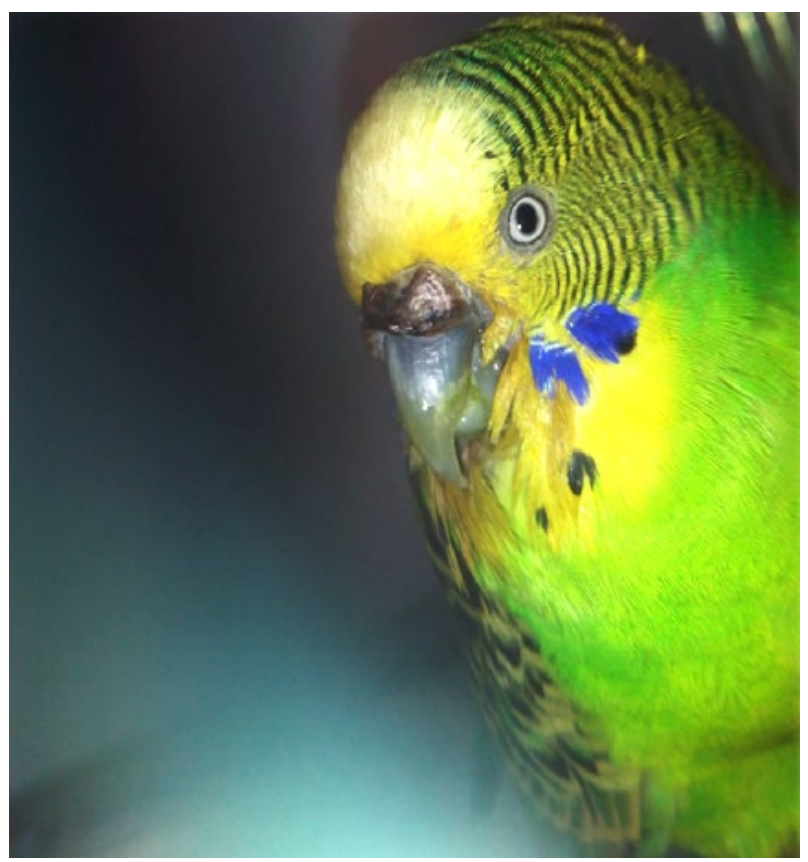

Figura 1. Periquito australiano (Melopsittacus undulatus) macho de siete años de edad. En el examen físico general, se encontró hipertrofia y cera de color café (interpretada como cambio de coloración de la cera).

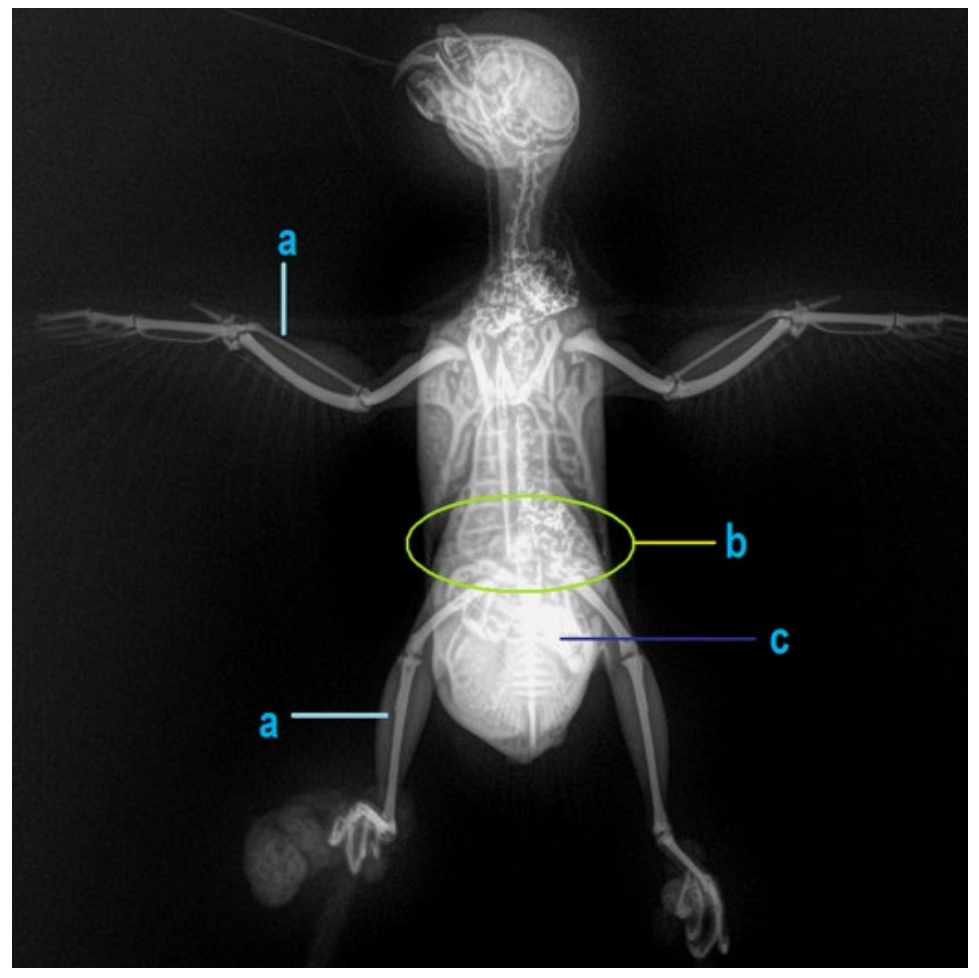

Figura 2. Imagen radiográfica ventrodorsal: a) la zona medular de todos los huesos largos se encuentra ocupada por un tejido radiopaco interpretado como hueso medular de nueva formación; b) tejido de nueva formación en la zona hepatogonado-renal; c) desplazamiento visceral. 


\begin{tabular}{|c|c|c|c|}
\hline MEDICAMENTO & DOSIS & VIA & FRECUENCIA \\
\hline $\begin{array}{c}\text { Solución de lactato de sodio compuesto (solución } \\
\text { Hartmann inyectable PISA) }\end{array}$ & $\begin{array}{l}\text { Día 1: } 4.08 \mathrm{~mL} \text {, Días } 2 \text { y } 3 \text { : } \\
3.06 \mathrm{~mL} \text {, Mantenimiento: } 2.04 \mathrm{~mL}\end{array}$ & Subcutánea & BID (cada 12 horas) \\
\hline Tramadol & $30 \mathrm{mg} / \mathrm{kg}$ & Oral & BID (cada 12 horas) \\
\hline Lactulosa & $150 \mathrm{mg} / \mathrm{kg}$ & Oral & BID (cada 12 horas) \\
\hline Vitamina $\mathrm{E}$ & $0.1 \mathrm{~mL} / \mathrm{kg}$ & Oral & SID (cada 24 horas) \\
\hline Omega 3 & $0.11 \mathrm{~mL} / \mathrm{kg}$ & Oral & SID (cada 24 horas) \\
\hline
\end{tabular}

Los siguientes cuatro días desde el ingreso hospitalario, no se observó mejoría a pesar del tratamiento, seguía aumentando de peso, pero con la misma condición corporal (1/5), no se apreció diarrea y la disnea no remitió. Debido a la condición del ave, el propietario solicitó la eutanasia.

Se realizó la eutanasia con sobredosis de pentobarbital sódico a $1 \mathrm{~mL} / \mathrm{kg}$ vía intracelómica de acuerdo con la Norma Oficial Mexicana NOM-033-SAG/ZOO2014: Métodos para dar muerte a los animales domésticos y silvestres.

\section{Pruebas de laboratorio}

El ave fue enviada para estudios post mortem al Laboratorio de Diagnóstico e Investigación en Enfermedades de las Aves del Departamento de Medicina y Zootecnia de Aves de la FMVZ-UNAM.

\section{Necropsia}

La cera se observó moderadamente engrosada y color café oscuro. La región abdominal ventral se encontró severamente distendida con pérdida de plumas y la piel amarilla. Alrededor de la cloaca se apreció moderada cantidad de heces, uratos y material de color rojo, que fue interpretado como sangre. Su peso era de $50 \mathrm{gr}$ con condición corporal 1/5 (Figura 3).

Las asas intestinales se apreciaban severamente aumentadas de tamaño, distendidas y de color rojo (interpretado como congestión). El proventrículo y el ventrículo se habían desplazado al lado derecho del celoma. Los lóbulos renales estaban moderadamente aumentados de tamaño, de color café y con puntos de color blanco (interpretados como uratosis); asimismo, los uréteres contenían abundantes uratos.

Los testículos habían sido remplazados casi en su totalidad por un tejido de nueva formación adosado a la cara visceral de los riñones. Este tejido midió $2.8 \times 2 \times 1.4 \mathrm{~cm}$, bien delimitado y encapsulado, pesó $7.2 \mathrm{~g}$ (lo que representaba el $14 \%$ del peso corporal), era de consistencia firme y de color blanco a amarillo. La superficie de corte era lisa y amarilla. En una zona de la neoplasia se observó un quiste que contenía líquido de color rojo claro. 


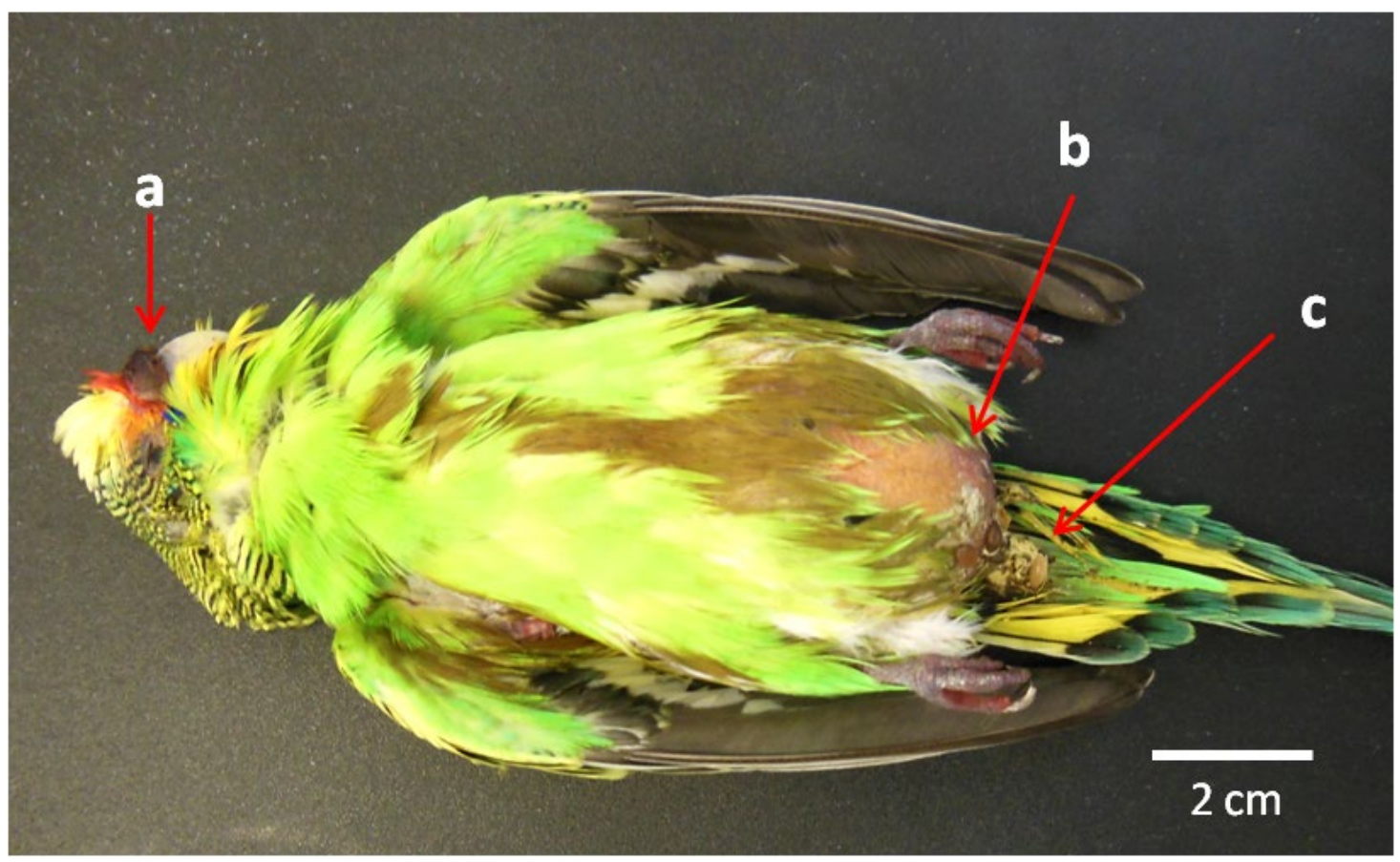

Figura 3. Fotomacroscópica del cadáver completo de un periquito australiano (Melopsittacus undulatus), en donde se observó hipertrofia y cambio de coloración de la cera (a), distensión abdominal severa y pérdida de plumas coberteras (b) y acumulación de heces alrededor del ano (c).

\section{Histopatología}

- Testículo: áreas hipercelulares que remplazaban la totalidad del parénquima testicular, áreas que estaban compuestas por células neoplásicas cúbicas a cilíndricas intratubulares separadas por moderada cantidad de tejido conectivo fibroso. Estas células se disponían formando palizadas con un núcleo redondo, hipercromático y moderada anisocariosis. El citoplasma era moderado, ligeramente eosinofílico y con algunas vacuolas en su interior (Figura 4). Se observó escasa cantidad de figuras mitósicas (0-1 por campo aleatorio de 40X).

- Hígado: los sinusoides hepáticos se exhibían dilatados y con abundantes eritrocitos mal conservados en su interior (congestión). También se observaron, de manera multifocal, grupos de hepatocitos intensamente eosinofilicos y con el núcleo picnótico y cariorrégico (necrosis coagulativa).

- Pulmón: los capilares y vasos sanguíneos contenían abundantes eritrocitos en su interior (interpretado como congestión) y, en la luz de los parabronquios y capilares aéreos, se apreció moderada cantidad de material eosinofilico homogéneo (interpretado como edema). En la periferia de los parabronquios se encontraron agregados de macrófagos con abundante cantidad de pigmento color negro (interpretado como antracosis).

- Tiroides: se encontraron grandes agregados de células inflamatorias compuestas principalmente por linfocitos bien diferenciados, así como, escasos folículos tiroideos. 


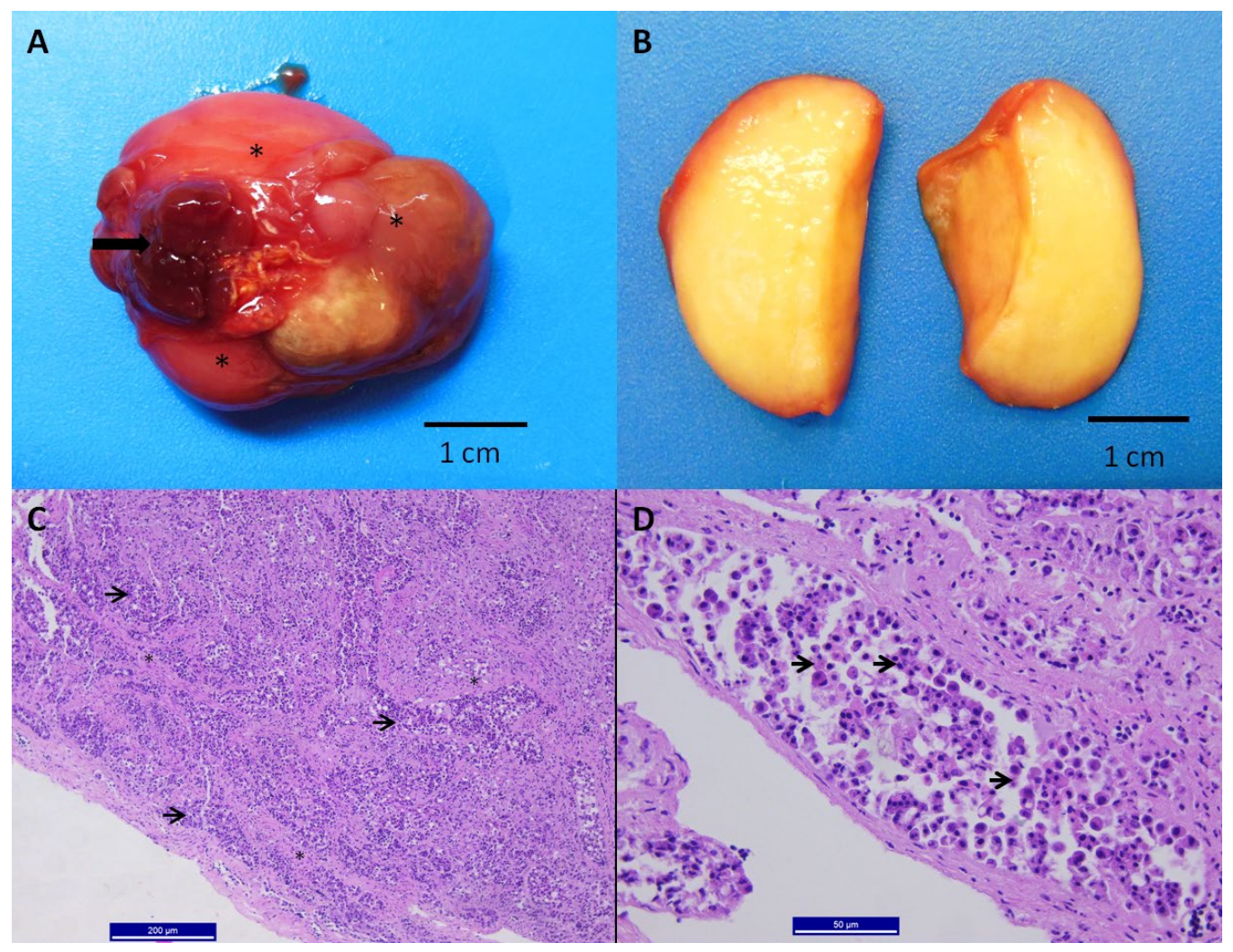

Figura 4. Tumor de células de Sertoli en un periquito australiano (Melopsittacus undulatus). (A) Tejido de nueva formación (asteriscos) bien delimitado y encapsulado, localizado en la región ventral de los riñones (flecha). (B) El tejido de nueva formación se encontraba bien delimitado, encapsulado, de consistencia firme, con la superficie de corte lisa y de color amarillo. (C) Fotomicrografía del tejido neoplásico, se observaron áreas hipercelulares (flechas) separadas por moderada cantidad de tejido conectivo fibroso (asteriscos). Tinción H\&E Barra $200 \mu \mathrm{m}$. (D) Se apreciaron células neoplásicas que se disponían en forma de palizada, de citoplasma moderado con escasas vacuolas, exhibían núcleo redondo, hipercromático y con moderada anisocariosis (flechas). Tinción H\&E Barra 50 m.

\section{Diagnósticos morfológicos microscópicos:}

1. Testículos: tumor de células de Sertoli

2. Hígado: hepatitis necrótica moderada multifocal coalescente aguda.

3. Pulmón: congestión y edema moderado difuso.

4. Tiroides: tiroiditis linfocítica moderada difusa con atrofia severa de los folículos tiroideos.

\section{Discusión y relevancia clínica}

En este caso clínico se demostró la presencia de un tumor de células de Sertoli en un periquito australiano (Melopsittacus undulatus) de siete años de edad. El tumor ocasionó hiperostosis y feminización. En esta especie se reporta una incidencia de neoplasias del 16.8 al $24.2 \%$ y en algunos estudios se menciona que el $69.7 \%$ de las neoplasias de psitácidos se diagnostican en periquitos australianos. ${ }^{1}$

Las neoplasias que se describen con más frecuencia en periquitos australianos son adenocarcinoma renal, neoplasias de tiroides, adenocarcinoma ovárico o del oviducto, tumores testiculares y lipomas. ${ }^{2}$ 
En un estudio realizado en 1203 periquitos australianos, se demostró que el $16.1 \%$ tenían neoplasias, de las cuales $23.2 \%$ fueron tumores renales, seguidas de lipomas (22.7\%), tumores gonadales (18\%) y tumores hepáticos $(13.4 \%)$. En algunos casos, los últimos dos tipos de tumores se asociaron a metástasis de los tumores renales. La mayor incidencia se observó entre el $3^{\text {er y }} 6^{\circ}$ año de vida. La claudicación y distensión abdominal fueron los signos clínicos predominantes para sospechar de un tumor abdominal, como ocurrió en este caso. ${ }^{3}$

Dentro de las neoplasias testiculares se incluyen seminomas, tumores de células intersticiales, linfomas, hemangiomas, fibrosarcomas, leiomiosarcomas y tumor de células de Sertoli; la mayoría de estas neoplasias se han reportado en periquitos australianos y no son comunes en otras aves. El tumor testicular más notable y el más fácil de diagnosticar es el de células de Sertoli. 4-6

El paciente cursó con signos clínicos de hiperestrogenismo; de acuerdo con la literatura, estos signos clínicos se explican de la siguiente manera: en los machos las células de Sertoli secretan niveles bajos de estrógenos; sin embargo, los tumores de células de Sertoli pueden ser funcionales, esto es, secretan muchos estrógenos e hiperestrogenismo.

Los signos clínicos que se describen en la literatura asociados a hiperestrogenismo son hernia abdominal por debilitamiento de los músculos abdominales, feminización en machos debido al exceso de estrógenos, que en los periquitos australianos se caracteriza por cambio de coloración en la cera, de azul a café, como ocurrió en este caso y, en otras especies de aves, se observa cambio en la coloración del plumaje; pancitopenia debido a que altos niveles de estrógenos provocan mielotoxicidad -especialmente en perros-.

Sin embargo, en este caso no fue posible demostrar esta alteración debido a que no se realizó un hemograma; hiperostosis poliostótica que se caracteriza por zonas radiopacas en la médula de los huesos, en las hembras es un proceso fisiológico que se asocia al estímulo de los estrógenos sobre las hormonas relacionadas con la formación de hueso y el desarrollo del hueso medular, que sirve como reserva de calcio para la formación del huevo en la época reproductiva y, algunos estudios sugieren que la hiperostosis poliostótica en machos es un hallazgo relacionado con el hiperestrogenismo como en este caso. 1, 4, 6-7

Otros signos clínicos asociados al tumor de células de Sertoli son anorexia, bradicardia, letargia, paresia unilateral o parálisis, disnea, distensión abdominal (desplazamiento visceral) y pérdida de peso; estos tres últimos también se observaron en nuestro paciente. La paresia unilateral o parálisis y la cianosis de miembros pélvicos, puede deberse a la compresión del nervio lumbosacro y de los vasos sanguíneos. ${ }^{5-6,8-9}$

El diagnóstico se realiza con base en los hallazgos del examen físico y de las placas radiografías en las que se observa una sombra alargada en la zona gonadorenal con desplazamiento caudoventral del aparato gastrointestinal e hiperostosis secundaria a hiperestrogenismo en todos los huesos (como se apreció en las radiografías de este caso).

Si la condición del paciente lo permite, se pueden realizar otras pruebas diagnósticas como ultrasonido, laparotomía y endoscopía en donde se vería el tumor. El diagnóstico definitivo se realiza mediante histopatología del tumor. 1,6,10

El tratamiento en este caso clínico se orientó a tratar los primeros diagnósticos presuntivos; debido a que el estado del paciente imposibilitó cualquier otra prue- 
ba diagnóstica. A pesar de ello, en la literatura se mencionan diferentes tipos de tratamientos.

La orquiectomía en tumores testiculares pequeños es factible y es el tratamiento de elección, aunque el pronóstico es de reservado a malo. Existen dos casos reportados en la literatura de aves con tumores en testículo, a las cuales se les realizó orquiectomía, una ninfa (Nymphicus hollandicus) y un periquito australiano (Melopsittacus undulatus), la primera murió durante la cirugía y, la segunda, en el periodo de recuperación. Mientras en otros dos casos el tumor fue completamente removido y las aves sobrevivieron tres años (Amazona aestiva aestiva) y 39 meses (Nymphicus hollandicus). ${ }^{8}$

Algunos tumores de células de Sertoli responden de manera paliativa a tratamientos con agonistas de $\mathrm{GnRH}$ (hormona liberadora de gonadotropinas). Los agonistas de $\mathrm{GnRH}$ sintéticos tienen alta afinidad con los receptores de $\mathrm{GnRH}$. Su acción agonística conducirá a un incremento en la expresión y liberación de gonadotropinas en la sangre, provocando un aumento en la producción de hormonas sexuales provenientes de la gónada.

A causa de que la GnRH endógena es de vida corta y es liberada en forma pulsátil; a la administración prolongada del agonista sintético de la GnRH (ya sea por aplicación de liberación continua o por repetición de la administración), el estímulo gonadotrópico inicial será remplazado por un prolongado efecto antigonadotrópico y en consecuencia la síntesis y liberación de la hormona gonadal se reduce. En casos donde el agonista de GnRH de larga acción es usado con la intención de regular el sistema reproductor, el efecto estimulatorio inicial, antes del prolongado efecto antigonadotrópico es preocupante y puede resultar en un empeoramiento inicial de los signos clínicos relacionado con el incremento en la producción de la hormona gonadal. ${ }^{1-12}$

Los agonistas de larga acción más evaluados en aves son el acetato de leuprolide y el acetato de deslorelina. ${ }^{12}$ El acetato de leuprolide de larga acción está disponible en diferentes formulaciones. La más usada en la medicina aviar es la de un mes de duración. Se reporta que su efecto se verifica en menos de cuatro semanas. En algunos casos las dosis tan altas como 1500 a $3500 \mu \mathrm{g} / \mathrm{kg}$ han sido usadas para el tratamiento paliativo de la neoplasia ovárica en ninfas. Dosis similares también han sido recomendadas para el tratamiento de macroorquidismo ocasionado por diversas etiologías. Los principales clínicos prefieren las dosis de 400 y $1000 \mu \mathrm{g} / \mathrm{kg}$. Se recomienda repetir la dosis cada $2-3$ semanas. ${ }^{12}$

Los implantes de acetato de deslorelina también se han usado para el tratamiento paliativo de los signos clínicos en nueve periquitos australianos (Melopsittacus undulatus) tentativamente diagnosticados con tumores gonadales productores de hormonas. Bajo anestesia general, se colocó un implante de $4.7 \mathrm{mg}$ de acetato de deslorelina subcutáneamente en el pliegue de la rodilla. Se reportó respuesta al tratamiento en siete de nueve periquitos, de días a cuatro semanas. Los signos clínicos se presentaron nuevamente en un promedio de 19 semanas después de la primera administración. Los implantes fueron administrados reiteradamente después de que los signos clínicos reaparecieran con una duración promedio de 20 semanas.

El tratamiento de tumor de células de Sertoli como diagnóstico presuntivo en periquitos australianos con implantes de acetato de deslorelina fue efectivo para 
tratar temporalmente los signos clínicos secundarios a la producción anormal de estrógenos y no se reportaron efectos secundarios significativos. ${ }^{12}$

En el presente caso se asume que el tejido de nueva formación comprimía los sacos aéreos, lo cual pudo ocasionar que el corazón intentará compensar la falta de oxigenación, generando cambios de presión sistémica, observados en la histopatología, como congestión hepática, edema y congestión pulmonar, situación que agravó la condición disneica del paciente. El tejido de nueva formación obstruyó y desplazó las vísceras; clínicamente esto se observó a través de la disnea y la retención de heces.

Otros hallazgos importantes en la histopatología fue la tiroiditis linfocitaria, que bien pudo estar asociada a la inflamación crónica con la que cursó el paciente, y la hiperplasia moderada en paratiroides relacionada con el hiperestrogenismo que provoca cambios importantes en el metabolismo del calcio. La hiperostosis y el cambio de coloración en cera también se asocian al hiperestrogenismo con el que cursaba el paciente.

Los tumores de células de Sertoli son en su mayoría neoplasias benignas de las que se recomienda la resección quirúrgica temprana, y tienen un pronóstico reservado. En los pacientes aviares, nos encontramos ante el reto del diagnóstico oportuno, debido a que generalmente los signos clínicos comienzan a presentarse cuando la neoplasia está muy desarrollada, entonces el procedimiento quirúrgico se vuelve riesgoso y de mal pronóstico, lo que nos llevaría únicamente a implementar un tratamiento paliativo con el único fin de dar calidad de vida al paciente, por ello, algunas veces se sugiere la eutanasia. 


\section{Financiamiento}

Este artículo fue financiado con los recursos de los autores.

\section{Agradecimientos}

Al Hospital de Aves y al Laboratorio de Diagnóstico e Investigación en Enfermedades de las Aves del Departamento de Medicina Veterinaria y Zootecnia de Aves DMZA- FMVZ-UNAM por el apoyo en la realización de este caso clínico.

Al técnico histotecnólogo Alfredo Amadeo Díaz Estrada por el procesamiento de las muestras.

\section{Conflictos de interés}

Los autores declaran no tener ningún conflicto de interés.

\section{Contribución de los autores}

MVZ Abril Castro y MC Juan Carlos Morales Luna participaron en el manejo clínico del paciente y en la redacción del artículo.

MC Félix D. Sánchez realizó la necropsia e histopatología y la redacción del artículo.

\section{Referencias}

1. Ritchie BW, Harrison GJ. Avian medicine: principles and application. Florida (US): Wingers Publishing; 1994.

2. Zehnder AM, Kent MS. Avian Neoplasia: Current Challenges and Future Directions. In: Proceedings of $32^{\text {nd }}$ annual Association Avian Veterinarian conference, Seattle, Washington, USA. 2011;27-35.

3. Neumann U, Kummerfeld N. Neoplasms in budgerigars (Melopsittacus undulatus): clinical, pathomorphological and serological findings with special consideration of kidney tumours. Avian Pathol. 1983;12(3):353-62.

4. Uetsuka K, Suzuki T, Doi K, Nunoya T. Malignant Sertoli cell tumor in a goose (Anser cygnoides domesticus). Avian Dis. 2012;56:781-5.

5. Razmyar J, Dezfoulian O, Shojadoost B, Masoudifard M, Peighambari SM. Sertoli cell tumor in a pigeon (Columba livia). J Avian Med Surg. 2005;19(4):286-8.

6. Turrel MJ, McMillan M, Paul-Murphy J. Diagnosis of tumors of companion birds i. AAV Today. 1987;1(3):109-16.

7. Albert A, Soler M. Hernia abdominal en un periquito común (Melopsittacus undulatus). Clin Vet Pequeños Anim. 2001;21(4):349-51.

8. Reavill D, Echols MS, Schmidt R. Testicular tumors of 54 birds and therapy in 6 cases. AAV Annu Conf. 2004;25:335-7.

9. Rosen LB. Avian reproductive disorders. J Exot Pet Med. 2012;21(2):124-31.

10. Matos R. Calcium metabolism in birds. Vet Clin Exotic Anim. 2008;1 1 (1):59-82.

11. Doneley B. Avian medicine and surgery in practice companion and aviary birds. 2a ed. Australia: CRC PRESS; 2016.

12. Mans $\mathrm{C}$, Pilny A. Use of $\mathrm{GnRH}$-agonists for medical management of reproductive disorders in birds. Vet Clin North Am Exot Anim Pract. 2014;17(1):23-33. 